\title{
HYBRID MODELING BASED ON SCSG-BR AND ORTHOPHOTO
}

\author{
Guoqing Zhou ${ }^{1}$, Yu Huang ${ }^{1}$, Tao Yue ${ }^{1, *}$, Xiaozhu $\mathrm{Li}^{1}$, Wei Huang ${ }^{1,2}$, Chaoshuang $\mathrm{He}^{1}$, Zhiliang Wu ${ }^{1}$ \\ 1. Guangxi Key Laboratory of Spatial Information and Geomatics, Guilin University of Technology, \\ No. 12 Jian'gan Road, Guilin, Guangxi, 541004, China- (gzhou,yuetao)@glut.edu.cn \\ 2. Department of Mechanical and Control Engineering, Guilin University of Technology, \\ No. 12 Jian'gan Road, Guilin, Guangxi 541004, China-102016477@glut.edu.cn
}

KEY WORDS: Urban building, Three-dimensional modelling, Spatial structure solid geometry (SCSG), Boundary representation (BR), Orthophoto

\begin{abstract}
With the development of digital city, digital applications are more and more widespread, while the urban buildings are more complex. Therefore, establishing an effective data model is the key to express urban building models accurately. In addition, the combination of 3D building model and remote sensing data become a trend to build digital city there are a large amount of data resulting in data redundancy. In order to solve the limitation of single modelling of constructive solid geometry (CSG), this paper presents a mixed modelling method based on SCSG-BR for urban buildings representation. On one hand, the improved CSG method, which is called as "Spatial CSG (SCSG)" representation method, is used to represent the exterior shape of urban buildings. On the other hand, the boundary representation (BR) method represents the topological relationship between geometric elements of urban building, in which the textures is considered as the attribute data of the wall and the roof of urban building. What's more, the method combined file database and relational database is used to manage the data of three-dimensional building model, which can decrease the complex processes in texture mapping. During the data processing, the least-squares algorithm with constraints is used to orthogonalize the building polygons and adjust the polygons topology to ensure the accuracy of the modelling data. Finally, this paper matches the urban building model with the corresponding orthophoto. This paper selects data of Denver, Colorado, USA to establish urban building realistic model. The results show that the SCSG-BR method can represent the topological relations of building more precisely. The organization and management of urban building model data reduce the redundancy of data and improve modelling speed. The combination of orthophoto and urban building model further strengthens the application in view analysis and spatial query, which enhance the scope of digital city applications.
\end{abstract}

\section{INTRODUCTION}

With the continuous development of digital city, the requirements of urban building modelling are getting higher and higher, and the complexity of building shapes leads to great difficulties in modelling complex urban buildings accurately. Therefore, it is necessary to establish an effective data model to represent the spatial topological relations of complex buildings correctly. Under the requirement of the development of digital earth, to express the three-dimensional world precisely can not only achieve an immersive feeling, but also benefit the data query and analysis of digital cities. So, the combination of the building model with real texture and the remote sensing image meets the requirements of the development of the digital city and makes more sense to the three-dimensional modelling.

Scholars at home and abroad have done a lot of research on 3D modelling of buildings. CC-Modeler, a topological generator was designed, which fitted the plane and corrected the least square by correcting the probability relaxation equation, finally obtained the building model (Gruen A, 1998). A grammardriven modelling approach was used to rebuild a complete building based on the model shape grammar (Mathias M, 2011). The city tree was treated as the data structure for urban $3 \mathrm{D}$ model. Create a floor plan firstly, then simplify the floor plan, build a cluster, create a city tree, and visualize it to create a city 3D model finally (Mao B, 2011). An integrated system of GIS and CG was proposed to automatically generate $3 \mathrm{D}$ building models for building polygons (building footprints) and automatically generate roof-shaped of 3D building models through the calculation of skeleton lines. (Sugihara K, 2012). A three-step strategy for constructing a three-dimensional model of a building which simplifying the smallest side of buildings firstly and then using P-tree convex hull technology to carry out plane aggregation and reconstructing the building generalization model finally (Baig S U, 2013). Sasaki N discussed how to construct a building model by computing a network polygon model from a given set of Facetons models (Sasaki N, 2013). Sugihara K automatically generated 3D building models by constructing polygon rectification. However, the article creates three-dimensional building models that assume polygons are orthogonal and not suitable for nonorthogonal polygons (Sugihara K, 2015). An improved constructive solid geometry (CSG) modelling method--voxel growth method, is proposed; and a hybrid modelling method based on CSG and boundary representation (BR) is proposed by WANG J S. The CSG model was used to express the external shape, the BR is used to express the internal relations. (Wang $\mathrm{J}$ S, 2010).

In terms of data integration, Zhou G was presented a method, which integrates image knowledge and LiDAR point cloud data for urban digital terrain model (DTM) and digital building model (DBM) generation. The DBM is an Object-Oriented data structure, each polygon represents a roof surface of building (Zhou G, 2004). In 2006, a system was designed for visualizing $3 \mathrm{D}$ city models in a web explorer environment under the

\footnotetext{
${ }^{*}$ Corresponding author: Tao Yue;
}

E-mail: yuetao@glut.edu.cn. 
supports of the digital building model, the digital terrain model, large-scale true urban orthoimage, and actual building sidewall pictures (Zhou G, 2006). In 2014, Zhou G was developed a seamless fusion between LiDAR and aerial imagery on the basis of aspect graphs, which utilize the features of houses, such as geometry, structures, and shapes. The projections of 3D primitives, standing for houses, are represented by the aspects. In the aspect graph, the note represents the face aspect and the arc is described by attributes obtained by the formulated coding regulations, and the coregistration between the aspect and LiDAR data is implemented. The aspects and/or the aspect graph are interpreted for the extraction of houses, and then the houses are fitted using a planar equation for creating a digital building model (DBM) (Zhou G, 2014).

In summary, people have encountered bottlenecks in the modelling of 3D urban buildings in the past ten years due to the lack of accuracy and efficiency in modelling. At this stage the three-dimensional model looks very realistic, but the data in the model is different from the actual data, resulting in unreliable data analysis and decision-making basis. The existing methods for representing buildings have become more and more mature. Most domestic and foreign scholars express building data based on CSG models and use existing software to directly model or code modelling. However, there are still many problems such as large amount of data, storage redundancy and other issues, this is still a long way from the requirements of digital cities and smart cities.

In order to accurately establish complex urban building models with real texture images, a hybrid modelling method based on SCSG-BR for complex urban buildings is proposed. The SCSG$\mathrm{BR}$ model express the topological relationship of the complex building. The method combined file database and relational database is used to manage the data of three-dimensional building model that treat the texture information as attribute information of a surface element; the building model ID and texture ID are managed by relational database. The texture is loaded and stored when the 3D building model is loaded and stored, which changes the traditional method of transferring that the texture images data store in the computer according to the specified file name read the memory area and then texture mapping to the complex surface of the building surface by the texture mapping function. Our method improves the loading speed of texture images. During the data processing, the leastsquares algorithm with constraints is used to orthogonalize the building polygons and adjust the polygons topology to ensure the accuracy of the modelling data. Finally, this paper combines the building model with real texture and the corresponding orthophoto to realize the modelling and operation of spatial information query and analysis in digital city.

\section{HYBRID MODELING PRINCIPLE}

\subsection{Hybrid model of urban building based on SCSG-BR}

Urban building modelling can be seen as complex CSG modelling which is the decomposition and assembly of voxels. However, there is no spatial relationship between voxels, and the CSG model cannot represent the positional relationship between the faces and edges of the entity. Although the threedimensional model represented by the CSG tree is unique, there are many CSG trees can represent the same three-dimensional model. Therefore, an improved CSG modelling method is proposed, which is called "Spatial CSG (SCSG)" representation method. The SCSG-BR method use the dimensionally extended
Nine-Intersection model (DIM-9IM) to express the topological relations between voxels and determine the unique SCSG tree of urban building correctly and quickly.

The SCSG representation method determines the topological relationships between the three-dimensional building body elements firstly. As shown in Figure 1 (a), for complex urban buildings, the body can be decomposed into simple entities A, B, C, D, E and F. However, the relative positions of the simple voxels cannot be determined and the unique CSG tree cannot be determined. At this point, this paper according to DIM-9IM to determine the spatial relationship between simple entities (Figure 1).

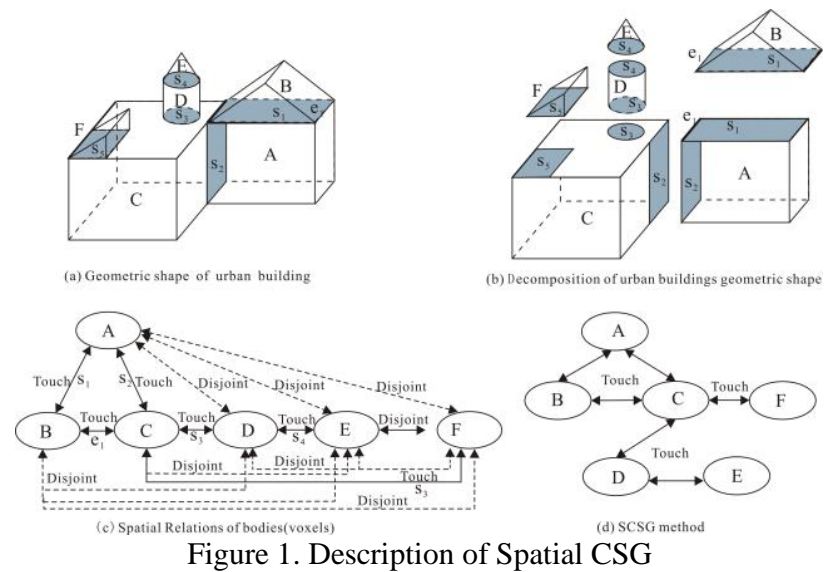

As shown in Figure 1, the geometric shape of urban building (Figure 1 (a)) and the decomposition of urban building (Figure 1 (b)) are shown. Starting from any simple voxel (assume from A), the voxels (B and C, A and B are connected to the surface $s$, and $\mathrm{A}$ and $\mathrm{C}$ are connected to the surface $s_{2}$ ) are analyzed to form a spatial topological relationship, and then from B or C to determine the spatial topological relations with the remaining simple voxels, and so on. The remaining voxels with a spatial relationship as follow: $\mathrm{B}$ and $\mathrm{C}$ are connected to $e_{1}$; $\mathrm{C}$ is connected to D by $s 3$ while $\mathrm{C}$ also connected to $\mathrm{F}$ by $s 5 ; \mathrm{D}$ is connected $\mathrm{E}$ by $s 4$. After the conclusion of the establishment of the spatial relationship between voxels, the relationship of voxels of the complex three-dimensional body (Figure 1 (c)) is determined, than the space CSG representation (Figure 1 (d)) is determined and the basic shape of body is determined.

The SCSG method can determine the unique shape of a complex urban building, but cannot express the spatial elements of urban building, such as the topological relationship between points, lines, faces and bodies. Therefore, the paper uses the boundary representation (BR) to describe the elements of urban 3D buildings. The BR method, which is represented by point, edge, ring and surface. It uses many curved surfaces or polygons to enclose and build an object entity. Many points are aggregated into edges while many edges are combined to establish a ring. Many rings are closed to establish a curved surface. The SCSG and BR combined to represent the shapes and topological relationships of the urban building models. Outside the urban building model, a SCSG tree is used to store voxels while the BR is used internally to represent detailed information about edges and points, which is equivalent to adding edges and points to a SCSG tree. In the hybrid model, the SCSG structure plays a decisive role while the BR structure as an auxiliary representation method. The SCSG-BR method reduces the complexity of the modelling process and can 
represent the geometry, topology and other information of the building model in detail.

\subsection{Data organization and storage}

Based on the SCSG-BR modelling method for complex urban buildings, the file database and relational database are combined to manage the data of urban building model which decrease the complex processes in texture mapping. The relational database stores attribute information of the building model and the texture; the file database contains a model file and a texture image file for storing the building model and the texture image. The data of urban building model contains spatial data and attribute data. Spatial data can be divided into geometric elements, three-dimensional objects and voxels. The geometric elements are linked to the attribute information to obtain a three-dimensional object and there is a strict topological relationship between the three-dimensional objects. Several three-dimensional objects establish a voxel, many simple building voxels establish a complex building. The attribute data contains the tables of attribute information about spatial elements, the raster images and texture information. The tables of attribute information are attached to the elements to generate three-dimensional object with topological relation. The texture information of raster images is attached to the face element, which is equivalent to see the texture information as an attribute of the face element. Finally, the urban texture model with real texture is combined with the corresponding orthophoto to obtain the realistic model (Figure 2).

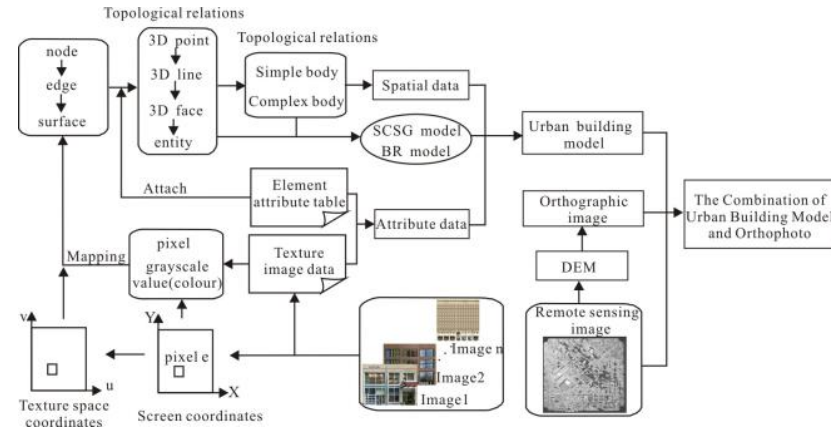

Figure 2. The data organization process of urban building model

According to the SCSG-BR modelling method for complex urban buildings, the data is managed by file database and relational database to manage all building model information. Relational database for storing building model and texture attribute information; the relationship between the forms are set according to ID; file database contains the model file, texture image files and remote sensing data files. The data is stored as Figure 3 shown.

As shown in Figure 3, the data tables include a 3D city building model table, a table of points, a line table, a face table, a voxel table, a wall texture table and a roof texture table. This tables are associated by setting IDs; file data includes a model file, texture image file and remote sensing image file. The model file is storing the urban model. The texture image file is for storing the acquired texture images. A remote sensing image file is stored for the corresponding orthophoto of the building model and the DEM data extracted from the remote sensing image. Building models are stored in OBJ format. Texture images data contains the raster image data of the wall texture and the roof texture, unified as a JPG format. The texture data is stored in a texture form in the relational database by a variable-length binary data type, OBLOB field type. An image texture can be stored in a record.

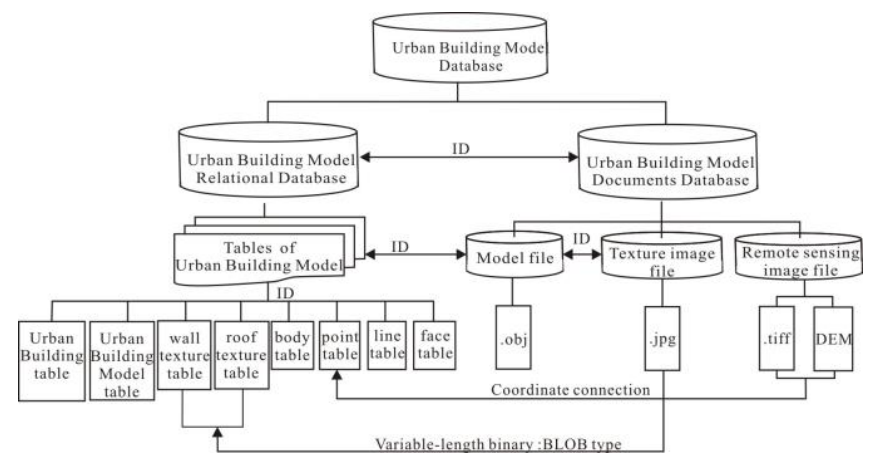

Figure 3. The storage of urban building data

\subsection{Modelling process}

According to the idea of hybrid modelling method and the combination of the orthophoto and DEM data, this modelling process can be divided into several parts (Figure 4).

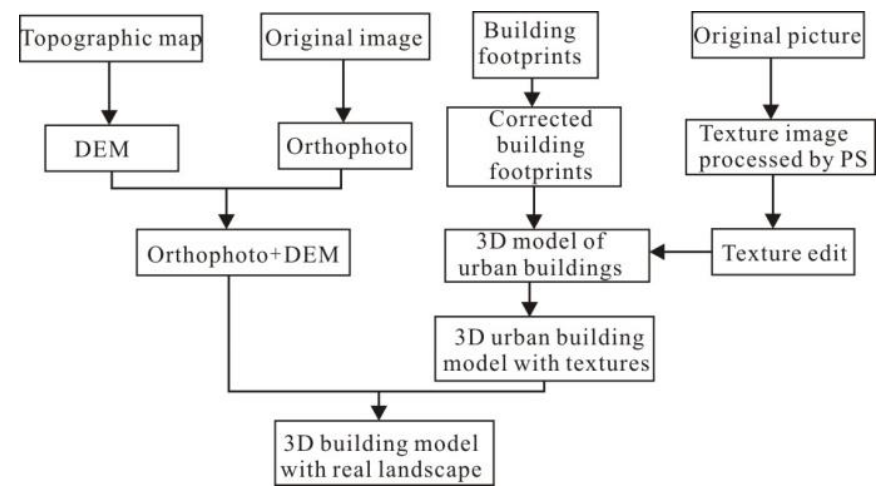

Figure 4. Modelling process

In Figure 4, in the order from left to right, top to bottom, the modelling process as follow:

(1) The original image is processed in ArcGIS to obtain the orthophoto, which is combined with the DEM data to obtain the combined image.

(2) With the least squares method with constraints, the original data of buildings (building footprints) are normalized and topologically adjusted in matlab.

(3) If the two-dimensional data is building footprint, attach height value to surface data than stretch the footprint by the height value. In the case of three-dimensional data, the closed surface data is modelled as a three-dimensional solid model (SCSG).

(4) Use the Photoshop software to process building texture images.

(5) Texture up the 3D white building model to get the 3D building model with real texture.

(6) Combine urban building model with orthophoto and DEM data in VRML.

\section{EXPERIMENT}

\subsection{Data source}

In order to reflect the extensive use of the data representation method in this article, this paper selects Denver, Colorado, USA 
as the study area to establish urban building realistic model. The density of buildings in this area is relatively large, and the structure of buildings is rather complex. Using these data to make 3D urban buildings model can better reflect the modelling method in this paper. The DBM spatial data obtained by DSM (Digital Surface Modelling), which contains only the threedimensional information of a building, including planar coordinates and roof removal information (Zhou G, 2005).

\subsection{Experimental process and result}

3.2.1 Data preprocessing: Orthogonalization and topology adjustment are operated for building data (building footprints) in matlab. Using Photoshop tools to modify the texture data; georeferencing of remote sensing images in ERDAS and then generating digital elevation model (DEM) for later data integration.

3.2.2 Building a 3D building model and texture mapping: Buildings are divided into simple buildings and complex buildings. Simple buildings can be represented by simple voxels, and complex buildings are composed of simple voxels. In the process of composition, topological relations between simple voxels and complex voxels are formed. Boolean operations are used to form complex buildings with correct shape, and the real images are added to the surface elements as the texture information to obtain the real sense of city buildings model.

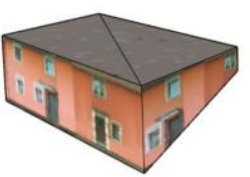

(a) (b)

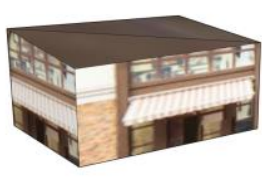

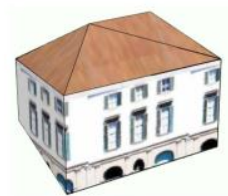

(c)
Figure 5. Construction of simple buildings

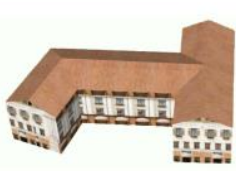

(a)

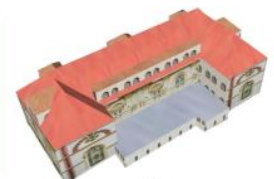

(b)

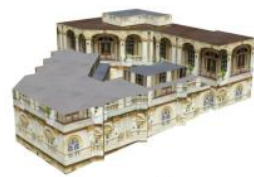

(c)
Figure 6.Complex building models

3.2.3 The combination of 3D urban building model and orthophoto with DEM: In ERDAS, import the established DEM into the Virtual GIS viewer window and do a simple exaggeration of the terrain that set the exaggeration to 1.5 ; and then re-create the model layer by Model Library to select and import the established model of urban buildings. After the model is imported into the scene, the size, position and orientation of the model are adjusted, models are linked and attributes are established to facilitate future space query. When the model is imported, due to the impact of the terrain and the location of the elevation and height of the model, an appropriate position needs to be adjusted so that the model cannot be completely placed. The combination of the 3D building model and orthophoto with DEM is shown in Figure 7.

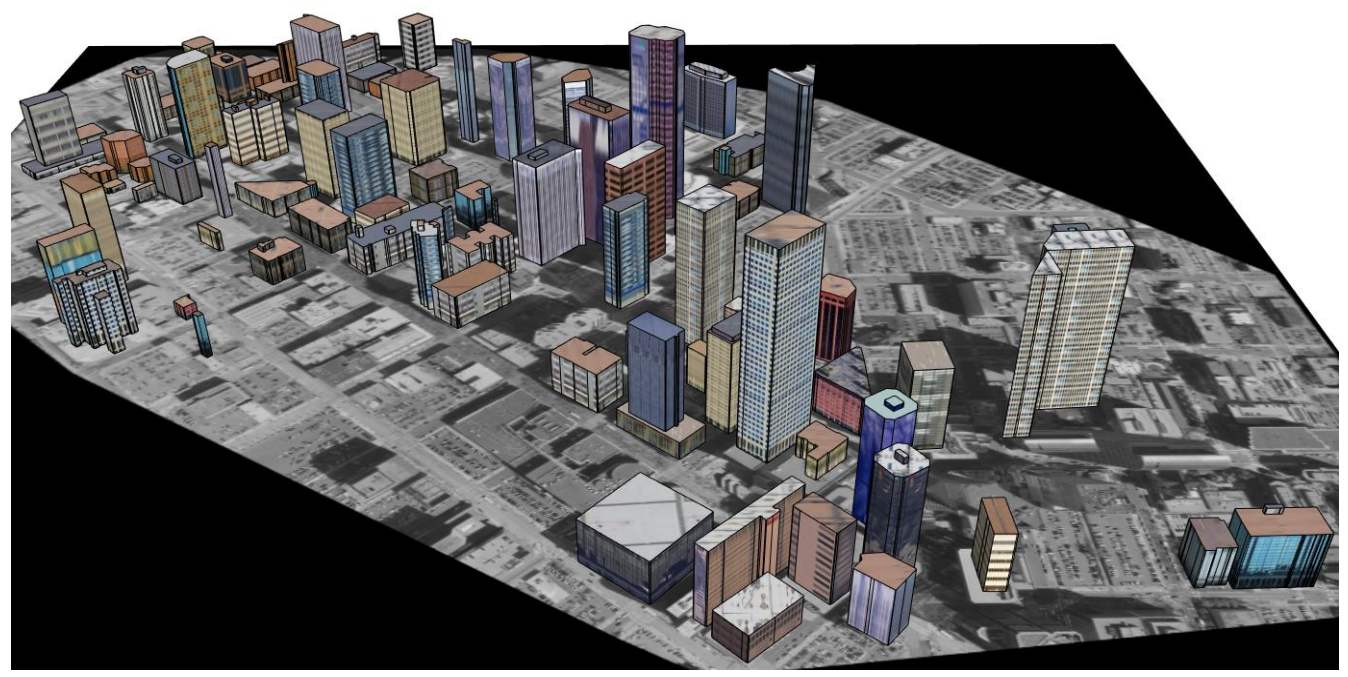

Figure 7. Combination of urban building model and orthophoto

In Figure 7, according to the hybrid model and data organization of this paper, the SCSG tree is used to store voxels of three-dimensional building model so that shape of building can be represented correctly; according to the topological relationship between voxels, the unique SCSG tree can be quickly identified, the time of traversal the voxels and the modelling can be reduced; internally, the BR is used to represent the detailed information of edges and points, which can make up for the lack of relationship between internal topologies and CSG. The BR representation with texture sees the texture as the attribute information of the surface element, and changes the traditional texture image invocation. SCSG and BR with textures learn from each other and quickly build a realistic 3D urban buildings model. At the same time, the combination of 3D building model and orthophoto has a real and beautiful effect, which is conducive to the operation of spatial analysis and spatial query.

\section{CONCLUSION}

In the past ten years, people have encountered bottlenecks in the modelling of 3D urban buildings in the past ten years due to the lack of accuracy and efficiency in modelling. At this stage the three-dimensional model looks very realistic, but the data in the model is different from the actual data, resulting in unreliable data analysis and decision-making basis. In this paper, the SCSG-BR representation method can more accurately represent 
the entity's topological relationship. On the basis of this, a method of storing textures and model data at the same time is proposed. The texture is treated as the attribute data of the wall and the roof surface. The 3D building model is managed by the method combined file database and relational database, which can decrease the complex processes in texture mapping. And, the combination of orthophotos and building models in ERDAS further strengthens the application in view analysis and spatial query, which enhance the scope of digital city applications.

\section{ACKNOWLEDGEMENTS}

This paper is financially supported by the National Natural Science of China under Grant numbers 41431179, the National Key Research and Development Program of China under Grant numbers 2016YFB0502500, the State Oceanic Administration under Grant numbers 2014\#58, GuangXi Natural Science Foundation under Grant numbers 2015GXNSFDA139032, Guangxi Science \& Technology Development Program under the Contract numbers GuiKeHe 14123001-4 and GuangXi Key Laboratory of Spatial Information and Geomatics Program under Grant numbers 151400701, 151400712, and 163802512.

\section{REFERENCES}

Baig S U, Rahman A A, 2013. A three-step strategy for generalization of 3D building models based on City GML specifications. Geojournal, 78(6), pp.1013-1020.

Gruen A, Wang X, 1998. CC-Modeler: a topology generator for 3-D city models1. ISPRS Journal of Photogrammetry \& Remote Sensing, 53(5), pp. 286-295.

Ledoux H, Meijers M, 2011. Topologically consistent 3D city models obtained by extrusion. International Journal of Geographical Information Science, 25(4), pp. 557-574.

Mathias M, Martinovic A, Weissenberg J, and Gool L V, 2011. Procedural 3D Building Reconstruction Using Shape Grammars and Detectors. International Conference on 3D Imaging, Modeling, Processing, Visualization and Transmission. IEEE, pp. 304-311.

Mao B, Ban Y, Harrie L, 2011. A multiple representation data structure for dynamic visualization of generalized 3D city models. ISPRS Journal of Photogrammetry \& Remote Sensing, 66(2), pp. 198-208.

Sugihara K, 2012. Knowledge-based automatic generation of 3D building models from building footprint by straight skeleton computation. International Journal of Knowledge and Web Intelligence, 3(4), pp. 361-371.

Sasaki N, Chen H T, Sakamoto D, and Igarashi T, 2013. Facetons: Face primitives for building 3D architectural models in virtual environments. ACM Symposium on Virtual Reality Software and Technology. ACM, pp. 77-82.

Sugihara K, Shen Z J, 2015. Automatic Generation of 3D Building Models by Rectification of Building Polygons. Advanced Science Letters, 21(12), pp. 3649-3654.

Wang J S, Gu W J, 2010. A three-dimensional hybrid modeling method of single building. International Conference on Future Information Technology and Management Engineering. IEEE, pp. 326-329.

Zhou G, Song C, Simmers J, and Cheng P, 2004. Urban 3D GIS from LiDAR and digital aerial images. Computers and Geosciences, 30(4), pp. 345-353.

Zhou G, Tan Z, Cen M, and Li C, 2006. Customizing visualization in Three-Dimensional urban GIS via Web-Based Interaction. Journal of Urban Planning and Development, 132(2), pp. 97-103.

Zhou G, Chen W, Kelmelis J A, and Zhang D, 2005. A comprehensive study on urban true orthorectification. IEEE Transactions on Geoscience and Remote Sensing, 43(9), pp. 2138-2147.

Zhou G, Zhou X, 2014. Seamless Fusion of LiDAR and Aerial Imagery for Building Extraction. IEEE Transactions on Geoscience and Remote Sensing, 52 (11), pp. 7393 - 7407. 